\title{
The Impact of Resistanceand Aerobic Exerciseson Blood Sugar Level in Type 2 Diabetes Mellitus: Apilot Study
}

\author{
Kapil Upadhyay $^{1}$, Rituraj Verma ${ }^{2}$, Taruna Verma ${ }^{3}$ \\ ${ }^{1}$ Student Researcher, BPT Galgotias University, Greater Noida, ${ }^{2}$ Associate Prof., Physiotherapy HOD, MPT \\ (Orthopedics), Galgotias University, Greater Noida, ${ }^{3}$ Assistant Prof., Galgotias University, Greater Noida
}

\begin{abstract}
Objective: To rule out theimpact of resistanceand aerobic exerciseson sugar level in the blood in type 2 diabetes mellitus population.

Sample: 10 subjects who have type 2 diabetes mellitus comes under this study.

Method: 10 subjects having type 2 diabetes mellitus were taken to control and experimental sets. Experimental set received aerobic and resistance exercise training program as well as medication while control set received only medication for 4 weeks ( 6 weeks per day).

Results: Paired t-test was used, and it was found that blood sugar was reduced in experimental set and two sample t-test was used to check between the group analyses and between set comparison it was found that there was no significant difference detected in sugar level in the blood.

Conclusion: It was concluded that resistanceand aerobic exercises are beneficial for lowering the blood glucose level in type 2 diabetes mellitus population.
\end{abstract}

Keywords: Type 2 diabetes mellitus (T2 DM), Aerobic exercise, Resistance exercise.

\section{Introduction}

Daily physical activities are necessary to maintain blood sugar in type 2 diabetic population ${ }^{[1]}$.

Resistance exercise and aerobic exercise reduces the risk factors to develop cardio vascular diseases, obesity and help to maintain overall health. Aerobic exercises involves large muscle group's movement which depend on large amount of energy produced by aerobic activities that is running or swimming ${ }^{[2]}$.

Resistance exercise is physical workout that causes the muscles to contract against an external resistance,

\footnotetext{
Corresponding Author:

Dr. Rituraj Verma

Associate Prof., Physiotherapy HOD, MPT

(Orthopedics), Galgotias University, Greater Noida

e-mail: rituraj.verma@galgotiasuniversity.edu.in

Mobile No.: 9953434095
}

the aim of that is to increase muscle strength and muscle $\operatorname{mass}^{[3]}$.

Diabetes is a condition in which blood sugar level of the body become impaired due to inadequate insulin secretion from the pancreas or poor sensitivity of the body towards insulin ${ }^{[4]}$.

Type 2 diabetes is distinguish by reduced insulin production in blood compared to the blood glucose level with peripheral insulin resistance, which results in chronic hyperglycemia. Type 2 diabetes is categorized by three fundamental metabolic abnormalities:

1. Impaired insulin production by glucose stimulation in the pancreas.

2. Major organ system like muscle, liver, adipose tissue become less sensitive to the insulin

3. Hepatic glucose produces in more abundance in the basal state.

Diabetes mellitus may result in complications. Diabetes is associated with accelerated atherosclerosis 
formation which leads to acute myocardial infarction. In addition, diabetes can cause retinopathy, nephropathy, autonomic neuropathy and cerebral stroke. The life expectancy of diabetes patient is reduced to two-thirds that of a non-diabetic individual. Diabetes 1 and 2 are diagnosed by detection of a fasting ( $>8$ hours) plasma glucose level which exceed by $126 \mathrm{mg} / \mathrm{dL}$, or blood sugar level more than $200 \mathrm{mg} / \mathrm{dL}$ at 2 hours after an oral glucose tolerance test ${ }^{[5]}$.

Component of physical activity in the form of physical workout or sports is necessary or important thing to manage the diabetes because of its effects in reducing blood glucose levels. The principle of exercise for diabetics is not different from other sport. The exercise aims to burn calories, convert glucose into energy, so the sugar in the blood would decrease.Currently, it is calculable that around one hundred fifty million people in the world suffer from diabetes mellitus, and it'll most likely double by 2025 . The causes of rising diabetes mellitus sufferers in developing countries are increasing population, aging, unhealthy diets, obesity and lack of activities. Physical activity for 3-4 times every week, minimum time for 75 minutes a week. The exercise aims to burn calories, convert glucose into energy, so the sugar in blood would decrease ${ }^{[6]}$.

Peoples with obesity are under the risk to develop diabetes mellitus. By involving in regular physical workout or activity, individuals can reduce excessive body weight and by controlling and maintaining wholesome diet, individuals can control type 2 diabetes mellitus and prevent the problem from developing where they must take ordinary insulin tablets ${ }^{[7]}$.

\section{Methodology}

Sampling: A sample of convenience was chosen for the study.

Sample Setting: A written consent form of patient and the inclusion criteria were considered the basis of their participation in the study.

Sample Size: A sample of 10 patients was taken for the study.

\section{Inclusion Criteria:}

- Age 35-60 years

- Population with type 2 diabetes mellitus.

\section{Exclusion Criteria:}

- Age below 35 years

- Type 1 diabetes

- Subjects should not have any musculoskeletal condition, cardiovascular condition or any neurological condition.

\section{Instrumentation:}

- Glucometer

Protocol: A total number of 10 subjects aged 3560 years were selected based on inclusion criteria and with a written consent form of patient to participate in the study. After completion of the consent form, body weight, body height, pre fasting and post prandial blood sugar levels was measured.We divided the subjects into twosets that is experimental set and control set. Experimental set consisting of 5 subjects received both types of exercise as well as medications while controlled group consisting of 5 subjects received medications only for 4 weeks ( 6 days per week). The control set will receive medications only. After completion of 4 weeks exercise training program post fasting and postprandial blood sugar levels was measuredby using glucometer.

\begin{tabular}{|l|l|}
\hline Aerobic Exercise & Resistance Exercise \\
\hline & $\begin{array}{l}\text { Resistance band exercise: 30 minutes } \\
\text { daily exercise with Thera-band for 6 days } \\
\text { in a week. } \\
\text { Upper body exercises training program: } \\
\text { biceps curl, push-ups, overhead triceps, } \\
\text { Walking: A } \\
\text { 30-minutes daily red row, shoulder lateral raise, forward } \\
\text { walk for 6 days in } \\
\text { a week } \\
\text { raise were included. } \\
\text { Lower body exercises training program: } \\
\text { squats, leg extensions, leg curl, hip } \\
\text { extension, standing abduction were } \\
\text { included. }\end{array}$ \\
\hline
\end{tabular}

Data Analysis: Analysis were done for 10 subjects to complete the study. The physical characteristics data of the subject including age, height, weight and BMI that descriptive summarized. Paired t test performed within the group analysis. Two sample $t$ test was used to check between the group analyses. Graphical illustration was done using MS-EXCEL 2016.

\section{Results}

The study involved 10 patients of type 2 diabetic mellitus aged from 35 to 60 years. The patients were separated into two equal sets: the first group did aerobic and resistance exercise as well as medications for 4 weeks ( 6 days per week). The second group took medications only for 4 weeks. Then we compared the 
effect of boththe exercises on the blood sugar of the patient.

By this experiment we found that there is no significant changes in the data (Table 1). The average age of the subjects in experimental set was $52 \pm 5.43$ and average age of control set was $48 \pm 3.50$ with $t$ value 1.17 and $p$ value 0.27 .

Table 1. Physical Characteristics of Subjects

\begin{tabular}{|l|c|c|c|c|}
\hline Variable & Experimental set $(\mathbf{n}=\mathbf{5})($ Mean \pm SD) & Control set $(\mathbf{n}=\mathbf{5})(\mathbf{M e a n} \pm$ SD) & T-value & P-value \\
\hline Age $($ Year) & $52 \pm 5.43$ & $48.6 \pm 3.50$ & 1.17 & 0.27 \\
\hline Height $(\mathrm{m})$ & $1.688 \pm 0.04$ & $1.694 \pm 0.09$ & -0.12 & 0.90 \\
\hline Weight $(\mathrm{kg})$ & $67.6 \pm 5.94$ & $69.6 \pm 6.18$ & -0.52 & 0.61 \\
\hline BMI $(\mathrm{kg} / \mathrm{m}$ & $23.72 \pm 1.73$ & $24.36 \pm 2.59$ & -0.45 & 0.65 \\
\hline
\end{tabular}

Intragroup comparison of experimental set, it was found that blood glucose level demonstrated statistically significant difference between PRE FBS level and PREPP sugar level and PO.FBS leveland PO. PP sugar level. On comparison, after exercise blood sugar level was improved (Table 2).

Table 2. Intragroup Comparison of Blood Glucose Level of Experimental Set

\begin{tabular}{|l|c|c|}
\hline Pair wise comparison & T value & P value \\
\hline PRE FBS and PRE-PP & 4.07 & $0.0152^{*}$ \\
\hline PO.FBS and PO.PP & 4.08 & $0.015^{*}$ \\
\hline
\end{tabular}

PR.FBS- pre fasting blood sugar, PO.FBS- post fasting blood sugar. PR. PP- pre post-prandial, PO. PP- post post-prandial. ${ }^{*} \mathrm{P}<0.05$, statistical significance.

On the other, on intragroup comparison of control set, the result shows no significant difference observed between PRE FBS level and PRE-PP glucose leveland PO.FBS level and PO. PP glucose level (Table 3). On comparison, after exercise blood sugar level was improved.

Table 3. Intragroup Comparison of Blood Glucose Level of Controlled Set

\begin{tabular}{|l|c|c|}
\hline Pair wise comparison & T value & P value \\
\hline PRE FBS and PRE-PP & 1.61 & 0.181 \\
\hline PO.FBS and PO.PP & 2.37 & 0.076 \\
\hline
\end{tabular}

PRE FBS- pre fasting blood sugar, PO.FBS- post fasting blood sugar

PRE-PP- pre post-prandial, PO. PP- post post-prandial

Though, on between set comparison it was found that there was no statistical differencedetected in sugar level in the blood. The average values of fasting and postprandial blood sugar level were significantly decreased from $148.4 \pm 33.45$ and $225.8 \pm 65.85$ to $128.4 \pm 33.65$ and $206.4 \pm 51.42$, respectively, in experimental set and from $157 \pm 31.53$ and $186.44 \pm 18.41$ to $154.6 \pm 28.77$ and $182.6 \pm 17.99$, respectively, in control set (Table 4). So, it is concluded that with the help of aerobic andresistance exercise on the daily basis, the blood sugar level of the body reduced in T2 DM.

\section{Discussion}

In this research, we studied the impact of resistance and aerobic exercise on the daily basis on sugar level in the blood in T2 DM population. The physical demographics variables taken were age, weight, height and BMI. The results suggested that, on comparing the pre and post data in experimental group, result shows that after aerobic and resistance training for 4 weeks helps in reduction of blood sugar level (Table 2). On the other hand, there was the reduction in controlled group also but on comparison between experimental and controlled group, better results show in the experimental group. So, it is concluded that by doing regular physical exercise the blood glucose level reduced in the body in T2 DM population.

It was understood from one of the discoursephysical workout can control the blood glucose and helpful for overall health improvement ${ }^{[2]}$.Another study examined that organized exercise of more than 2 hours and 30 minutes per week shows a better reduction compare to the exercise of exact 150 minutes or less than 150 minutes per week ${ }^{[i]}$. As reported by Rajiv Sighamoney et al., 2018 investigated that before exercise training program the mean value blood glucose level was $143.937 \mathrm{mg} / \mathrm{dl}$ 
and after exercise was $112.1 \mathrm{mg} / \mathrm{dl}$ and the mean value of post prandial blood sugar before the exercise training program was $193.167 \mathrm{mg} / \mathrm{dl}$ and after the exercise was $156.49 \mathrm{mg} / \mathrm{dl}$. So, they concluded that aerobic exercise improves the activity of insulin in blood, which result in the lowering of blood sugar in T2 DM population ${ }^{[4]}$.

The present study indicates that by doing aerobics and resistance exercise regularly is beneficial for lowering the blood sugar level in the type 2 diabetic population. Another research suggest that aerobic and resistance exercise is usually suggested for the care in the T2 DM population ${ }^{[3]}$.One of the researchersfound a major difference in blood sugar of the body in the intervention group with a value of $p<0.05$, which means there is a significant influence of diabetes exercises on the blood sugar levels ${ }^{[6]}$. Another review research by Elizabeth Anderson et al., 2018 suggested that by indulging in regular physical workout is helpful in the elimination and management of chronic diseases. They concluded that by indulging in regular physical activity can provide a non-invasive mode of preventing the longterm chronic diseases and provide numerous health benefits, improve or encourage societal growth and improves overall universal health ${ }^{[8]}$.

Resistance and aerobic exercise help to ameliorate the action of insulin and maintain the level of glucose or lipids in blood. It is also beneficial in maintaining blood pressure and improve the quality of living ${ }^{[9]}$. According to PeeyooshaGurudut et al., in 2017 concluded that passive static stretching, and resistance exercises are evenly advantageous in lowering the blood glucose level and helpful for the management of glycemic control ${ }^{[10]}$.

In general, by indulging in regular physical exercise or any sports activity is useful for the lowering the blood glucose level in type 2 diabetic patients. Our findings suggest that, both the sets (experimental set and controlled set) show reduction in blood sugar level. However, the experimental set shows better result than the controlled set. So, it is attributed that aerobic and resistance exercise helps in lowering the blood sugar level. Though, on between set evaluation no statistical difference was detected for blood glucose level. In experimental set, an average difference of $14 \mathrm{mg} / \mathrm{dL}$ was detectedamong the pre- and post-post-prandial period and $20 \mathrm{mg} / \mathrm{dL}$ was detectedamong the pre- and post-fasting period suggested that aerobic and resistance exercise helpful in lowering the blood glucose level. In contrast, in controlled set, an average difference of $4 \mathrm{mg}$ /
$\mathrm{dL}$ and $3 \mathrm{mg} / \mathrm{dL}$ was found during the fasting and postprandial period (Table 4).

Future Research: Future study is needed to see long term effect of resistance and aerobic exercise daily in type 2 diabetic patient. The future research could be done by comparing several activities or exercise and their impact in the population of T2 DM.

\section{Conclusion}

In order to get the result of resistance and aerobic exercises on sugar level in the blood in type 2 diabetic people, this research was held. It is found that, there is a significant difference in blood sugar of the body before and after the aerobic and resistance exercise in postprandial period in the experimental set with a value of $\mathrm{p}<0.05$. And by this study we concluded that resistance and aerobic exercise is beneficial for lowering the glucose level in blood T2 DM people.

Ethical Clearance: Participants gave informed consent before taking part.

\section{Source of Funding: Self}

\section{Conflict of Interest: Nil}

\section{References}

1. Way KL, Hackett DA, Baker MK, Johnson NA. The effect of regular exercise on insulin sensitivity in type 2 diabetes mellitus: a systematic review and meta-analysis. Diabetes \& metabolism journal. 2016 Aug 1;40(4):253-71.

2. Colberg SR, Sigal RJ, Yardley JE, Riddell MC, Dunstan DW, Dempsey PC, Horton ES, Castorino K, Tate DF. Physical activity/exercise and diabetes: a position statement of the American Diabetes Association. Diabetes care. 2016 Nov 1;39(11):2065-79.

3. Wang T, Liu Y, Zhong R, Xu D, Wang H, Fu BS. Benefit effects of aerobic exercise and resistance training on the management of type 2 diabetes. Int J Clin Exp Med. 2018 Jan 1;11(10):10433-45.

4. Sighamoney R, Jadhav V, Yeole U. Effect of aerobic exercises on diabetes mellitus in young adults.

5. Brukner P. Brukner \& Khan's clinical sports medicine. North Ryde: McGraw-Hill; 2012.

6. Mustikawati D, Erawati E, Supriyatno H. Effect Of The Diabetes Exercise On The Blood Sugar Levels 
In Diabetes Mellitus Patients. Journal of Nursing Care. 2020 Mar 19;3(1).

7. Bhagat U, Singh A. Effect of diabetes on daily life and role of yogic activities on diabetes. Int. J. Yoga. 2017;2(2):338-40.

8. Anderson E, Durstine JL. Physical activity, exercise, and chronic diseases: A brief review. Sports Medicine and Health Science. 2019 Dec 1;1(1):3-10.

9. Colberg SR, Sigal RJ, Fernhall B, Regensteiner JG, Blissmer BJ, Rubin RR, Chasan-Taber L, Albright AL, Braun B. Exercise and type 2 diabetes: the American College of Sports Medicine and the American Diabetes Association: joint position statement executive summary. Diabetes care. 2010 Dec 1;33(12):2692-6.

10. Gurudut P, Rajan AP. Immediate effect of passive static stretching versus resistance exercises on postprandial blood sugar levels in type 2 diabetes mellitus: a randomized clinical trial. Journal of exercise rehabilitation. 2017 Oct;13(5):581.

11. Smith AD, Crippa A, Woodcock J, Brage S. Physical activity and incident type 2 diabetes mellitus: a systematic review and dose-response meta-analysis of prospective cohort studies.

12. Hamasaki H. Daily physical activity and type 2 diabetes: a review. World journal of diabetes. 2016 Jun 25;7(12):243.

13. Regensteiner JG, Bauer TA, Huebschmann AG, Herlache L, Weinberger HD, Wolfel EE, Reusch JE. Sex differences in the effects of type 2 diabetes on exercise performance. Medicine and science in sports and exercise. 2015 Jan;47(1):58.

14. Zhao RR, O'Sullivan AJ, Singh MA. Exercise or physical activity and cognitive function in adults with type 2 diabetes, insulin resistance or impaired glucose tolerance: a systematic review. European Review of Aging and Physical Activity. 2018 Dec 1;15(1):1. 